PROCEEDINGS OF THE

AMERICAN MATHEMATICAL SOCIETY

Volume 129, Number 5, Pages 1359-1363

S $0002-9939(00) 05662-8$

Article electronically published on October 20, 2000

\title{
ON THE DUNFORD-PETTIS PROPERTY OF THE TENSOR PRODUCT OF $C(K)$ SPACES
}

\author{
FERNANDO BOMBAL AND IGNACIO VILLANUEVA
}

(Communicated by Dale Alspach)

\begin{abstract}
In this paper we characterize those compact Hausdorff spaces $K$ such that $C(K) \hat{\otimes} C(K)$ (and $C(K) \hat{\otimes}_{s} C(K)$ ) have the Dunford-Pettis Property, answering thus in the negative a question posed by Castillo and González who asked if $\ell_{\infty} \hat{\otimes} \ell_{\infty}$ and $C[0,1] \hat{\otimes} C[0,1]$ have this property.
\end{abstract}

\section{INTRODUCTION}

In [7] it is asked if $\ell_{\infty} \hat{\otimes} \ell_{\infty}$ and $C[0,1] \hat{\otimes} C[0,1]$ have the Dunford-Pettis Property (DPP). The purpose of this paper is to show that this is not the case, and to characterize those $C(K)$ spaces such that $\hat{\otimes}_{n} C(K)$ or $\hat{\otimes}_{n, s} C(K)$ have the DunfordPettis Property, when $n>1$.

Let us recall that a Banach space $E$ has the DPP if, for any Banach space $F$, every weakly compact operator from $E$ into $F$ is completely continuous. In this paper we will need the following facts about the DPP; they are all well known, and can be found, for instance, in [9]: (a) $C(K)$ spaces and Schur spaces have the DPP, (b) if a dual Banach space $E^{*}$ has the DPP, then so does $E$ (but the reciprocal is not true), (c) the DPP is stable under complemented subspaces.

We will now explain our notation. Throughout the paper $K$ will denote a compact Hausdorff space and $C(K)$ the space of continuous scalar functions defined on it. We will write $\mathcal{L}(E ; X)$ to indicate the linear operators from $E$ into $X$, and $\mathcal{L}^{n}\left(E_{1}, \ldots, E_{n} ; X\right)$ will denote the space of continuous multilinear operators from $E_{1} \times \cdots \times E_{n}$ into $X$. When $E_{1}=\cdots=E_{n}=E$ we will write this as $\mathcal{L}^{n}(E ; X)$ and in this case $\mathcal{L}_{s}^{n}(E ; X)$ will refer to those of the previous operators which are symmetric. In all the cases, if $X=\mathbb{K}$, the scalar field, we will not write it. We will write the projective tensor product of $E_{1}, \ldots, E_{n}$ as $E_{1} \hat{\otimes} \cdots \hat{\otimes} E_{n}$. We will write $\hat{\otimes}_{n} E$ to denote the $n$-fold projective tensor product of $E$, and $\hat{\otimes}_{n, s} E$ will denote the symmetric $n$-fold projective tensor product of $E$. If $n=2$, we will sometimes also write this as $E \hat{\otimes}_{s} E$.

We consider to be well known that $\mathcal{L}^{n}\left(E_{1}, \ldots, E_{n} ; X\right)$ is naturally isometric to $\mathcal{L}\left(E_{1} \hat{\otimes} \cdots \hat{\otimes} E_{n} ; X\right)$ and that $\mathcal{L}_{s}^{n}(E ; X)$ is naturally isometric to $\mathcal{L}\left(\hat{\otimes}_{n, s} E ; X\right)$.

We will use the convention ${ }^{[i]}$. to indicate that the $i^{\text {th }}$ coordinate is not involved.

Received by the editors February 2, 1999 and, in revised form, July 20, 1999.

1991 Mathematics Subject Classification. Primary 46B28, 47B07.

Key words and phrases. Dunford-Pettis property, spaces of continuous functions, projective tensor product.

Both authors were partially supported by DGICYT grant PB97-0240.

(C)2000 American Mathematical Society 


\section{The RESUlts}

As is well known, every linear operator from any $C(K)$ space into the dual of another $C(K)$ space is weakly compact, and therefore completely continuous. Using that, we can prove the following lemma.

Lemma 2.1. Let $K_{1}, K_{2}$ be two compact Hausdorff spaces. Let $\left(f_{n}\right) \subset C\left(K_{1}\right)$ be a weakly null sequence and let $\left(g_{n}\right) \subset C\left(K_{2}\right)$ be a bounded sequence. Then the sequence $\left(f_{n} \otimes g_{n}\right) \subset C\left(K_{1}\right) \hat{\otimes} C\left(K_{2}\right)$ is weakly null.

Proof. Let $\phi \in\left(C\left(K_{1}\right) \hat{\otimes} C\left(K_{2}\right)\right)^{*}$, and let us consider the linear operator $S \in$ $\mathcal{L}\left(C\left(K_{1}\right) ; C\left(K_{2}\right)^{*}\right)$ associated to it defined by

$$
S(f)(g)=\phi(f \otimes g) .
$$

Let us suppose without loss of generality that $\sup _{n}\left\|g_{n}\right\| \leq 1$. Since $S$ is completely continuous, we get that

$$
\lim _{n \rightarrow \infty}\left|\phi\left(f_{n} \otimes g_{n}\right)\right| \leq \lim _{n \rightarrow \infty}\left\|S\left(f_{n}\right)\right\|=0 .
$$

In [4] it can be seen that, if $K$ is scattered, then $\left(\hat{\otimes}_{n} C(K)\right)^{*}$ and $\left(\hat{\otimes}_{n, s} C(K)\right)^{*}$ are Schur spaces for every $n \in \mathbb{N}$. With analogous proof it can be proved that, in case $K_{1}, \ldots, K_{n}$ are scattered, $\left(C\left(K_{1}\right) \hat{\otimes} \cdots \hat{\otimes} C\left(K_{n}\right)\right)^{*}$ is a Schur space.

We now state our main result.

Theorem 2.2. Let $K_{1}, K_{2}$ be two infinite compact Hausdorff spaces. Then $C\left(K_{1}\right)$ $\hat{\otimes} C\left(K_{2}\right)$ has the DPP if and only if both $K_{1}$ and $K_{2}$ are scattered.

Proof. If both $K_{1}$ and $K_{2}$ are scattered, then $\left(C\left(K_{1}\right) \hat{\otimes} C\left(K_{2}\right)\right)^{*}$ is a Schur space and therefore $C\left(K_{1}\right) \hat{\otimes} C\left(K_{2}\right)$ has the DPP. Now, let us suppose that one of them, say $K_{2}$, is not scattered. Since $K_{1}$ is infinite, $C\left(K_{1}\right)$ is not Schur, and therefore there exist two sequences $\left(f_{n}\right) \subset B_{C\left(K_{1}\right)}$ and $\left(\xi_{n}\right) \subset B_{\left(C\left(K_{1}\right)\right)^{*}}$ such that $\left(f_{n}\right)$ is weakly null and $\xi_{n}\left(f_{n}\right)=1$ for every $n \in \mathbb{N}$. Also, since $K_{2}$ is not scattered, $C\left(K_{2}\right)$ contains an isomorphic copy of $\ell_{1}$, and therefore there exists a continuous surjective operator $q: C\left(K_{2}\right) \mapsto \ell_{2}([10$, Corollary 4.16]). Then let us consider the trilinear form

$$
T: C\left(K_{1}\right) \times C\left(K_{2}\right) \times C\left(K_{2}\right) \mapsto \mathbb{K}
$$

defined by

$$
T(f, g, h)=\sum_{n=1}^{\infty} \xi_{n}(f) q(g)_{n} q(h)_{n}
$$

and let us consider the linear operator

$$
\hat{T}^{1}: C\left(K_{1}\right) \hat{\otimes} C\left(K_{2}\right) \mapsto\left(C\left(K_{2}\right)\right)^{*}
$$

canonically associated to it given by

$$
\hat{T}^{1}(f \otimes g)(h)=T(f, g, h) .
$$

It is clear that $\hat{T}^{1}=q^{*} \circ \psi \circ \phi$ where

$$
\phi: C\left(K_{1}\right) \hat{\otimes} C\left(K_{2}\right) \mapsto \ell_{2}
$$


is given by

$$
\phi(f \otimes g)=\left(\xi_{n}(f) q(g)_{n}\right)_{n},
$$

and $\psi \in \mathcal{L}\left(\ell_{2} ; \ell_{2}^{*}\right)$ is the canonical linear isometry identifying both spaces. Since $\psi$, $\phi$ and $q^{*}$ are all weakly compact, so is $\hat{T}^{1}$.

So we now just have to see that $\hat{T}^{1}$ is not completely continuous. Let us consider a sequence of bounded functions $\left(g_{n}\right)_{n} \subset C\left(K_{2}\right)$ such that $q\left(g_{n}\right)=e_{n}$, where $\left(e_{n}\right)$ is the canonical basis of $\ell_{2}$. Then, according to Lemma 2.1, the sequence $\left(f_{n} \otimes g_{n}\right)_{n} \subset C\left(K_{1}\right) \hat{\otimes} C\left(K_{2}\right)$ weakly converges to zero, but, for each $n \in \mathbb{N}$,

$$
\left\|\hat{T}^{1}\left(f_{n} \otimes g_{n}\right)\right\| \sup _{n}\left\|g_{n}\right\| \geq\left|\hat{T}^{1}\left(f_{n} \otimes g_{n}\right)\left(g_{n}\right)\right|=\left|T\left(f_{n}, g_{n}, g_{n}\right)\right|=1,
$$

a contradiction.

Remark 2.3. In [2], the following definition is stated: a multilinear form $T \in$ $\mathcal{L}^{n}\left(E_{1}, \ldots, E_{n}\right)$ is said to be regular if every one of the associated linear operators

$$
T_{1}^{i}: E_{i} \mapsto \mathcal{L}^{n-1}\left(E_{1}, .{ }^{[i]} ., E_{n}\right)
$$

is weakly compact, and this is shown to be equivalent to every one of the associated $(n-1)$-linear operators

$$
T_{n-1}^{i}: E_{1} \times \stackrel{[i]}{ } \cdot \times E_{n} \mapsto \mathcal{L}\left(E_{i}\right)
$$

being weakly compact. From the proof it is clear that, given an $i \in(1, \ldots, k), T_{1}^{i}$ is weakly compact if and only if $T_{n-1}^{i}$ is also, but there is no reason to believe that, for nonsymmetric multilinear forms, the fact that $T_{1}^{i}$ is weakly compact should imply that $T_{1}^{j}$ is weakly compact, too. The main idea behind our proof is to find a trilinear form such that (using the notation of [2]) $T_{1}^{1}$ is weakly compact but $T_{1}^{2}$ is not. In [11, a trilinear form on $\ell_{\infty}$ is used, which is a slight modification of a trilinear form defined in 1]. This form does exactly what we want it to do.

Remark 2.4. Although $\hat{T}^{1}$ is not completely continuous when considered as a linear operator, its bilinear counterpart

$$
T^{1}: C\left(K_{1}\right) \times C\left(K_{2}\right) \mapsto\left(C\left(K_{2}\right)\right)^{*}
$$

given by

$$
T^{1}(f, g)(h)=T(f, g, h)
$$

is completely continuous, according to the usual definition of completely continuous bilinear operator (i.e., if $\left(f_{1}^{n}\right) \subset C\left(K_{1}\right)$ and $\left(f_{2}^{n}\right) \subset C\left(K_{2}\right)$ are weakly Cauchy sequences, then $T^{1}\left(f_{1}^{n}, f_{2}^{n}\right)$ is a norm Cauchy sequence), as follows from [12] (indeed, it follows from [15] that every bilinear continuous operator from $C\left(K_{1}\right) \times C\left(K_{2}\right)$ into $\left(C\left(K_{2}\right)\right)^{*}$ is completely continuous). This proves a conjecture of [15] that states that the fact that a multilinear operator from the product of $C(K)$ spaces is completely continuous (considered as a multilinear mapping) does not imply that the same operator, when considered as a linear mapping from the projective tensor product of the spaces, has to be completely continuous.

It is well known that, for every Banach space $E_{1}, \ldots, E_{n}(n>1), E_{1} \hat{\otimes} \cdots \hat{\otimes} E_{n-1}$ is complemented in $E_{1} \hat{\otimes} \cdots \hat{\otimes} E_{n}$. Using this, the next corollary follows easily. 
Corollary 2.5. Let $K_{1}, \ldots, K_{n}$ be infinite compact Hausdorff spaces. Then $C\left(K_{1}\right)$ $\hat{\otimes} \cdots \hat{\otimes} C\left(K_{n}\right)$ has the DPP if and only if $K_{1}, \ldots, K_{n}$ are all scattered.

The theorem and corollary above remain true for the symmetric projective tensor product.

Theorem 2.6. Let $K$ be a compact Hausdorff space. Then $C(K) \hat{\otimes}_{s} C(K)$ has the $D P P$ if and only if $K$ is scattered.

Proof. If $K$ is scattered, then $\left(\hat{\otimes}_{n, s} C(K)\right)^{*}$ is a Schur space for every $n \in \mathbb{N}$, and therefore $C(K) \hat{\otimes}_{s} C(K)$ has the DPP. Now, if $K$ is not scattered, we can consider the trilinear form

$$
T: C(K) \times C(K) \times C(K) \mapsto \mathbb{K}
$$

defined by

$$
T(f, g, h)=\sum_{n=1}^{\infty} \frac{1}{2}\left(\xi_{n}(f) q(g)_{n}+\xi_{n}(g) q(f)_{n}\right) q(h)_{n}
$$

that is, the symmetrized respect to the two first variables of the trilinear form used in Theorem 2.2. Now we can apply analogous reasonings as before to conclude that the linear operator

$$
\hat{T}^{1}: C(K) \hat{\otimes} C(K) \mapsto(C(K))^{*}
$$

defined by

$$
\hat{T}^{1}(f, g)(h)=T(f, g, h)
$$

is weakly compact and not completely continuous.

Again, it is well known (see [3]) that, for every Banach space $E, \hat{\otimes}_{n-1, s} E$ is complemented in $\hat{\otimes}_{n, s} E$. So, the next corollary follows.

Corollary 2.7. Let $K$ be a compact Hausdorff space. Then, for every $n>1$, $\hat{\otimes}_{n, s} C(K)$ has the DPP if and only if $K$ is scattered.

As suggested by J. Gutiérrez, the next theorem can be proved with the same proof as above.

Theorem 2.8. Let $E$ be a Banach space such that

i) every linear operator from $E$ into $E^{*}$ is completely continuous,

ii) $E$ is not Schur, and

iii) $E$ has an isomorphic copy of $\ell_{1}$.

Then $E \hat{\otimes} E$ and $E \hat{\otimes}_{s} E$ do not have the DPP.

There are several spaces which are not $C(K)$ spaces and verify the conditions of the proposition above, among them, the space $H^{\infty}$ [5, [6], the disc algebra [8, 13], the space of analytic uniformly convergent Fourier series on the unit circle [14].

The authors thank Verónica Dimant and Joaquín Gutiérrez for some clarifying conversations. 


\section{REFERENCES}

[1] R. Aron, S. Y. Choi and J. Llavona, Estimates by polynomials, Bull. Austral. Math. Soc. 52 (1995), 475-486. MR 96k:46073

[2] R. Aron and P. Galindo, Weakly compact multilinear mappings, Proc. of the Edinburgh Math. Soc. 40 (1997), 181-192. MR 98e:46051

[3] F. Blasco, Complementation in spaces of symmetric tensor products and polynomials. Studia Mathematica 123 (2) (1997) 165-173. MR 98d:46043

[4] F. Bombal and I. Villanueva, Regular multilinear operators on $C(K)$ spaces. Bull. Austral. Math. Soc. 60 (1999), 11-20. CMP 99:17

[5] J. Bourgain, $H^{\infty}$ is a Grothendieck space, Studia Math. 75 (1983), 193-216. MR 85j:46092

[6] J. Bourgain, New Banach space properties of the disc algebra and $H^{\infty}$, Acta Math. 152 (1984), 1-48. MR 85j:46091

[7] J. Castillo and M. González, On the Dunford-Pettis Property. Acta Univ. Carol. Math. Phys. 35 (1994), 5-12. MR 97a:46018

[8] F. Delbaen, Weakly compact operators on the disc algebra, J. Algebra 45 (1977), 284-294. MR 58:2304

[9] J. Diestel, A survey of results related to the Dunford-Pettis property. Contemporary Math. 2 (1980), 15-60. MR 82i:46023

[10] J. Diestel, H. Jarchow and A. Tonge, Absolutely Summing Operators. Cambridge Univ. Press, 1995. MR 96i:46001

[11] M. González and J. M. Gutiérrez, Injective factorization of holomorphic mappings, Proc. Amer. Math. Soc. 127 (1999), 1715-1721. MR 99i:46033

[12] A. Pełczyński, A theorem of Dunford-Pettis type for polynomial operators. Bull. Acad. Polon. Sci. Ser. Sci. Math. Astr. Phys. 11 (1963), 379-386. MR 28:4370

[13] E. Saab and P. Saab, On stability problems of some properties in Banach spaces, in: K. Sarosz (ed.), Function Spaces, Lecture Notes Pure Appl. Math. 136, Dekker, New York 1992, 367-394. MR 92m:46021

[14] S. F. Saccone, The Pełczyński property for tight subspaces, J. Funct. Anal. 148 (1997), 86-116. MR 98i:46014

[15] I. Villanueva, Completely continuous multilinear operators on $C(K)$ spaces, to appear in Proc. Amer. Math. Soc. CMP 99:07

Departamento de Análisis Matemático, Facultad de Matemáticas, Universidad ComPlutense de Madrid, MAdrid 28040, Spain

E-mail address: bombal@eucmax.sim.ucm.es

Departamento de Análisis Matemático, Facultad de Matemáticas, Universidad ComPlutense de Madrid, Madrid 28040, Spain

E-mail address: ignacio_villanueva@mat.ucm.es 